\title{
Moyamoya Syndrome Associated with Mucolipidosis-II
}

\author{
P. de Guzman, M. Hamilton, A. Khan, M. Eesa, A. Kirton
}

Can J Neurol Sci. 2014; 41: 522-524

Moyamoya disease or moyamoya syndrome describes a progressive, non-inflammatory stenosis of the distal internal carotid arteries. This arteriopathy often presents early in life, and is an important cause of stroke and disability in children. Isolated moyamoya disease is most common in Asian populations where genetic explanations are emerging ${ }^{1}$. Moyamoya syndrome occurs within other conditions including sickle cell disease, Down syndrome, neurofibromatosis-1, cranial irradiation, and systemic vascular disorders such as pediatric fibromuscular dysplasia $^{2}$. The clinical presentation of pediatric moyamoya syndrome and stroke can be variable. Acute focal deficits secondary to large arterial strokes, gradual neurocognitive decline due to progressive watershed infarcts, and migraine-like headache disorders are possible. Neuroimaging with magnetic resonance imaging (MRI) can demonstrate distinctive patterns of hypoperfusion and injury. Angiographic modalities confirm arteriopathy extent and character while demonstrating the abnormal collaterals that produce the classical "puff of smoke" appearance.

Mucolipidosis-II (ML-II) and Mucolipidosis-III (ML-III) are autosomal recessive lysosomal storage disorders associated with reduced enzymatic function of UDP-N-acetylglucosamine: lysosomal hydrolase $\mathrm{N}$-acetylglucosamine-1-phosphotransferase (GlcNAc-PT), a product of the GNPTAB gene ${ }^{3}$. The phosphotransferase is essential to the synthesis of a mannose 6phosphate residue on lysosomal hydrolases, which targets trafficking of the enzymes to lysosomes. Deficiency of the phosphotransferase results in reduced levels of enzymes within the lysosome and accumulation of lysosomal enzymes outside the lysosome. Mucolipidosis-II, also known as I-cell disease, typically has an aggressive infantile onset and is often fatal in the first decade. Children typically present with early progressive developmental delay, coarse facial features, and multisystem disease including valvular insufficiency and orthopedic complications. Mucolipidosis-III is an attenuated form of ML-II with delayed onset and survival to 30 to 40 years-of-age and the two disorders exists along a continuum. The diagnosis of ML-II or ML-III requires evidence of low enzyme activity of GlcNAcPT, most often performed in cultured skin fibroblasts, and this can be associated with a mutation in the GNPTAB gene. Cerebrovascular complications in ML-II have not been described.

We report an eight year-old girl with ML-II diagnosed with moyamoya syndrome and successfully treated with revascularization surgery.

\section{Case Report}

An eight year-old left-handed girl was referred to our Pediatric Neurology Clinic for recurrent headaches. In the interim, screening MRI demonstrated cerebrovascular abnormalities and the referral was redirected to our Pediatric Stroke Clinic. Her past medical history was significant for MLII diagnosed at five years-of-age due to slowly progressive global developmental delay. Biochemical analysis identified a GNPTAB mutation (c.1030 $\mathrm{C}>\mathrm{T}$ ) with significantly reduced GlcNAc-PT enzymatic activity $(<3 \%)$ and elevated serum lysosomal hydrolases ( $\beta$-glucoronidase and $\beta$-galactosidase). Cognitive development was approximately equivalent to a five year-old but had been stable for several years. She was a happy and sociable child with moderate expressive language and articulation difficulties. She had severe gross motor delay, confined mostly to a wheelchair although she could ambulate short distances with a walker.

Headaches had been occurring for seven months with slowly increased frequency to approximately monthly. Intensity was moderate to severe and she would often cry and hold her head. Duration was protracted, typically lasting one to three days with frequent trips to the emergency room. Descriptions were limited by her development but pain appeared to be frontal, uni- or bilateral, with no apparent photophobia or sonophobia. Her appetite would decrease and she vomited on occasion. Use of non-prescription medications was inconsistent without clear efficacy. There were no associated focal neurological deficits suggestive of aura or ischemia. Family history was significant for migraine without aura in both parents and one brother, and consanguinity (parents are 1st cousins). There was no history of metabolic or cerebrovascular disorder.

On examination, the child had coarse facial features, normal vital signs including blood pressure, and mildly reduced growth (10th percentile for weight). Neurological exam revealed an alert

From the Calgary Pediatric Stroke Program, Section of Neurology (PdG, AKi);

Division of Neurosurgery, Department of Clinical Neurosciences (MH); Department of Medical Genetics and Pediatrics (AKh), Alberta Children's Hospital Research Institute;

Department of Radiology (ME), University of Calgary, Calgary, Alberta, Canada. Received November 4, 2013. Final Revisions Submitted February 27, 2014. Correspondence to: Adam Kirton, Section of Neurology, Alberta Children's Hospital, 2888 Shaganappi Trail NW, Calgary, AB, Canada T3B 6A8

Email: adam.kirton@albertahealthservices.ca. 


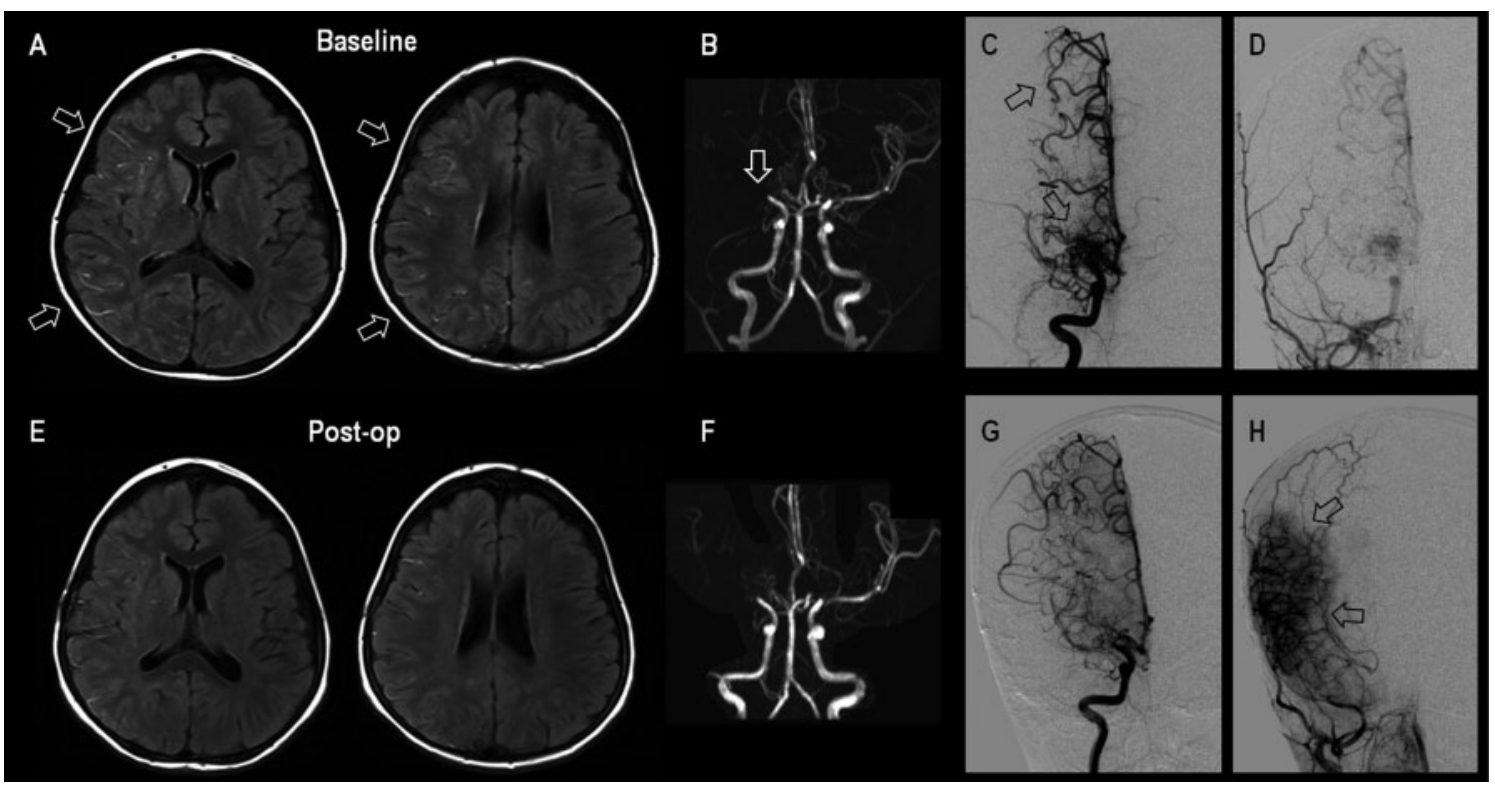

Figure: Neuroimaging at baseline (top row) and post-op (bottom row). (A) Original FLAIR MRI shows extensive sulcal hyperintensity ("Ivy sign") over right hemisphere. (B) MRA suggests distal internal carotid artery (ICA) occlusion. (C) Right ICA injection on cerebral angiogram confirms severe stenosis of distal ICA and middle cerebral artery with extensive perforating collaterals ("puff of smoke") and anterior cerebral artery leptomeningeal collaterals (arrow) supplying hypoperfused territories. (D) Right external carotid injection shows filling of a large superficial temporal artery and some reflux into the ICA. (E) Repeat FLAIR MRI at 12 months shows improvement in Ivy sign and no evidence of stroke. (F,G) MRA and right internal carotid artery injection are unchanged. $(H)$ External carotid injection now shows extensive angiogenesis into previously hypoperfused areas.

and sociable child with global delays consistent with the history above. She had dysarthric speech but otherwise intact cranial nerves. Motor exam demonstrated spastic quadriplegia, worse in the lower extremities with moderately reduced bulk and power, hyperreflexia with ankle clonus, upgoing plantar responses and mild contractures. She could stand and ambulate slowly with a walker. There was no evidence of sensory deficit or ataxia. Cardiovascular exam was normal including no cranial, carotid, or abdominal bruits.

Neuroimaging findings are summarized in the Figure. Initial MRI demonstrated normal brain parenchyma but fluidattenuated inversion recovery (FLAIR) sequences demonstrated abnormal linear sulcal hyperintensities throughout the right cerebral hemisphere consistent with impaired perfusion or socalled "Ivy sign". Magnetic resonance angiography (MRA) revealed severe stenosis or occlusion of the right distal internal carotid and middle cerebral arteries. The right proximal posterior cerebral artery also appeared stenotic. Subsequent conventional angiography confirmed the above arterial abnormalities and extensive abnormal deep collaterals consistent with moyamoya syndrome. No other laboratory investigations were planned. However, prior to surgery her partial thromboplastin time (PTT) was prolonged which led to the subsequent discovery of a positive lupus anticoagulant.

Our patient presented with a relatively attenuated form of ML-II, maintaining a high quality of life that was expected to continue for decades. However, due to her moyamoya syndrome, progressive headache disorder, and imaging markers of impaired perfusion, we considered her a high stroke risk. Following discussion between the family and experts in metabolics, pediatric stroke, and neurosurgery, it was agreed that the benefits of indirect revascularization surgery outweighed the low risk. A right-sided encephaloduroarteriosynangiosis (EDAS) was performed with branches of the superficial temporal artery transposed beneath the dura overlying frontal and parietal cortical areas with imaging evidence of impaired perfusion. The procedure was well tolerated without complication. Repeat cerebral angiography at 12 months demonstrated angiogenesis with new external carotid collaterals supplying previously hypoperfused brain (Figure 1). Repeat MRI at 12 months demonstrated no stroke, stability of moyamoya syndrome with no evidence of contralateral ICA disease, and improvement of the "Ivy sign" (Figure 1). At 18 months post-surgery her headaches were significantly reduced, being headache free for over six months. She is maintained on acetylsalicylic acid (ASA) (80mg OD) with stable neurological function.

\section{DISCUSSION}

We report a potential association between ML-II and moyamoya syndrome. Delay in diagnosis of pediatric moyamoya syndrome is common, creating a risk of both acute stroke as well as repeated subclinical infarctions leading to accumulated neurological dysfunction. Identification of associated conditions is therefore essential to identify at risk populations and screen them for stroke prevention given the ready availability of effective therapies. 
Our case highlights the utility of neuroimaging biomarkers in recognizing occult pediatric cerebrovascular disease. An MRA was included in this patient though the reasons are not clear and the original indication would likely not have prompted vascular imaging in many centres. Absence of MRA does not preclude identification of cerebrovascular disease, however. In our patient, imaging sequences of the parenchyma, such as a coronal T2, demonstrated large vessel flow voids (figure not shown) and stenosis within the right ICA/middle cerebral artery (MCA) (Figure 1). Another useful imaging biomarker is the Ivy sign, now well recognized to correlate with impaired cerebrovascular reserve in moyamoya syndrome ${ }^{4}$. Though a similar appearance can be seen under general anaesthesia, the asymmetrical pattern observed was a telling sign of large vessel arterial disease. Increased use and careful interpretation of these imaging modalities has the potential to enhance stroke prevention in children.

Surgical revascularization is highly effective with low complication rates and is currently recommended for children with moyamoya syndrome ${ }^{5}$. As the imaging biomarkers available with simple MRI (Ivy sign) are now well correlated with more advanced, higher risk testing such as acetazolamidesingle-photon emission computed tomography $(\mathrm{SPECT})^{4}$, the latter tests were not performed. Their presence suggested a high risk of future infarction while the risk of indirect revascularization was low. At 18 month follow-up, our patient was arguably asymptomatic; although, the marked improvement in her debilitating headaches seems attributable to surgery. The robust angiogenesis observed suggests that the underlying arteriopathic process present in our patient did not preclude this process from occurring. In contrast to the safe and efficacious interventions available for moyamoya syndrome, treatment options for ML-II are limited. Moyamoya syndrome may represent an under-recognized but treatable comorbidity of MLII. Given the neurodegenerative course of ML-II and ML-III, it is possible moyamoya syndrome or its consequences, such as white matter injury, could be missed or attributed to the disease itself on examination or imaging. Alternatively, moyamoya syndrome only complicates a small percentage of other genetic conditions (e.g. trisomy 21, neurofibromatosis-1) so the strength (or even existence) of a true association between ML-II and moyamoya syndrome cannot be proven by this single case.

The pathogenesis of moyamoya syndrome is poorly understood and multifactorial given the numerous ethnic, genetic, and syndromic associations. The improbability of our patient having two rare diseases suggests their ML-II played a causative role in their moyamoya syndrome. Arteriopathy has been reported in a single case of ML-II with proximal coronary artery abnormalities ${ }^{6}$, suggesting possible direct vascular effects. There are also at least 37 GNPTAB mutations identified, resulting in variable enzymatic defects, phenotypes, and perhaps cerebrovascular irregularities. Fabry disease is another lysosomal storage disorder that features cerebral arteriopathy with increased stroke risk $^{7}$. Intimal media thickening due to intracellular accumulation of globotriasylceramide (ganglioside) and lysoglobotriaosylsphingosine ${ }^{8}$ may mediate progressive vascular obstruction. Defining the organ-specific morbidities of lysosomal storage disorders influences diagnostic screening approaches while informing indications and outcomes for emerging therapies such as enzyme replacement. While not targeting the primary neurological and developmental complications of ML-II, prompt identification of cerebrovascular complications with early revascularization carries potential to prevent additional brain injury and morbidity.

If additional evidence beyond our single case can confirm an association between ML-II and moyamoya syndrome, screening MRA practices may need to be considered as they are at risk for other conditions (NF-1, Trisomy 21).

\section{REFERENCES}

1. Miyatake S, Miyake N, Touho H, et al. Homozygous c.14576G >A variant of RNF213 predicts early-onset and severe form of moyamoya disease. Neurology. 2012;78(11):803-10.

2. Scott RM, Smith ER. Moyamoya disease and moyamoya syndrome. N Engl J Med. 2009;360(12):1226-37.

3. Tiede S, Storch S, Lubke T, et al. Mucolipidosis II is caused by mutations in GNPTA encoding the alpha/beta GlcNAc-1phosphotransferase. Nat Med. 2005;11(10):1109-12.

4. Mori N, Mugikura S, Higano S, et al. The leptomeningeal "ivy sign" on fluid-attenuated inversion recovery MR imaging in Moyamoya disease: a sign of decreased cerebral vascular reserve? AJNR Am J Neuroradiol. 2009;30(5):930-5.

5. Roach ES, Golomb MR, Adams R, et al. Management of stroke in infants and children: a scientific statement from a Special Writing Group of the American Heart Association Stroke Council and the Council on Cardiovascular Disease in the Young. Stroke. 2008;39(9):2644-91.

6. Siles A, Mitchell GA, Dahdah NS. An infant with mucolipidosis-II and an atretic orifice of the left coronary artery. Cardiol Young. 2010;20(1):97-9.

7. Romao EA, Lourenco CM, Junior WM, et al. What lies beneath: Fabry nephropathy in a female patient with severe cerebrovascular disease. Clin Nephrol. 2013;79(4):330-4.

8. Aerts JM, Groener JE, Kuiper S, et al. Elevated globotriaosylsphingosine is a hallmark of Fabry disease. Proc Natl Acad Sci USA. 2008;105(8):2812-7. 